\title{
Correction to: Observations of a Radio-Quiet Solar Preflare
}

\author{
Arnold O. Benz ${ }^{1,2}$ (D) Marina Battaglia ${ }^{1}$ (D) \\ Manuel Güidel ${ }^{3}$
}

Published online: 5 February 2018

(c) Springer Science+Business Media B.V., part of Springer Nature 2018

\section{Correction to: Solar Phys \\ https://doi.org/10.1007/s11207-017-1175-3}

This article was published with an erroneous version of Figure 3. Please find in this document the correct version of the figure.

Combined Radio and Space-based Solar Observations: From Techniques to New Results Guest Editors: Eduard Kontar and Alexander Nindos

The online version of the original article can be found under https://doi.org/10.1007/s11207-017-1175-3.

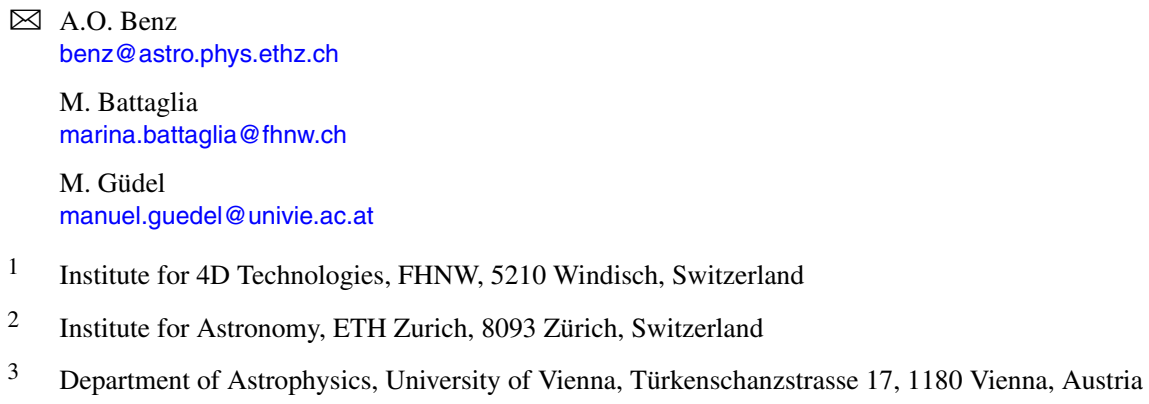

3 Department of Astrophysics, University of Vienna, Türkenschanzstrasse 17, 1180 Vienna, Austria 


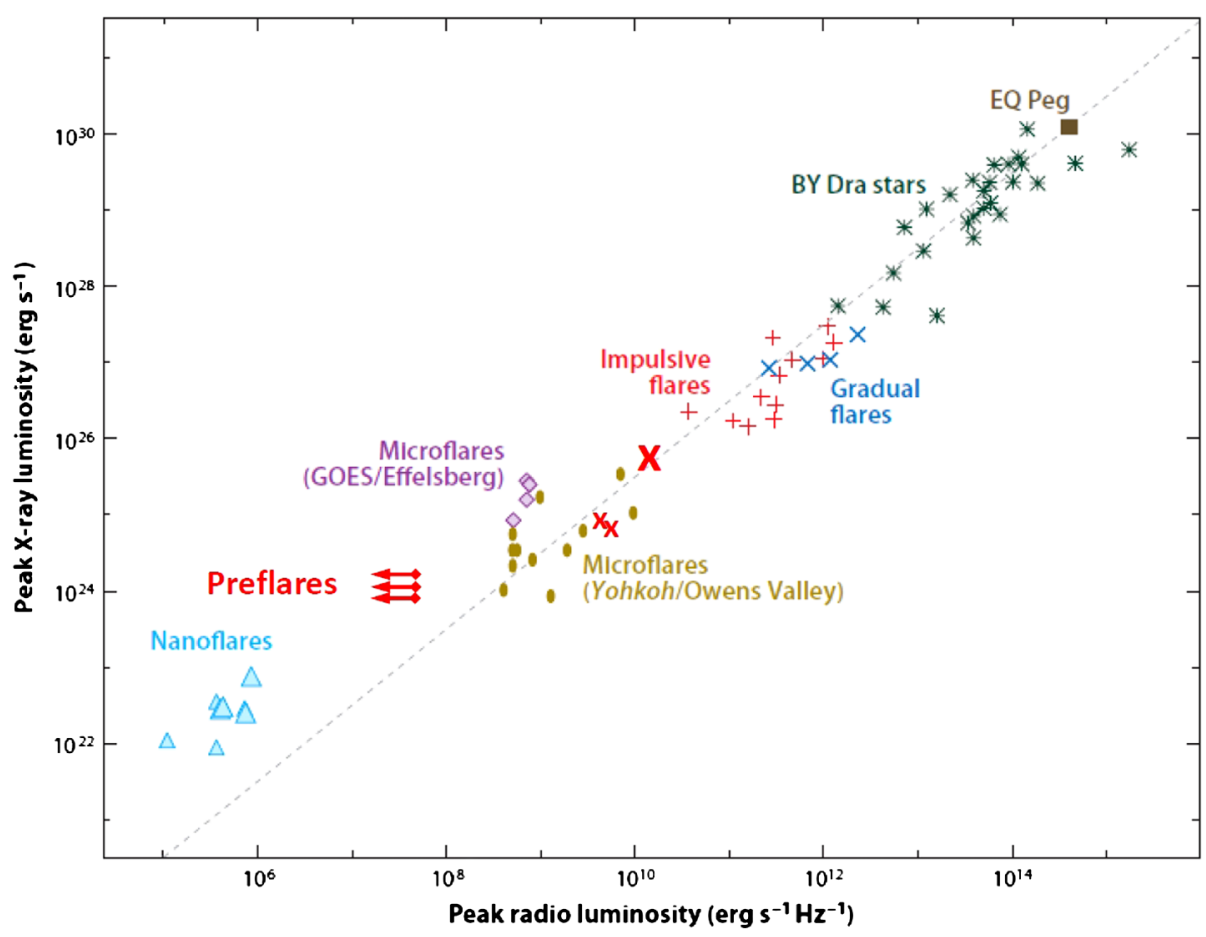

Figure 3 Comparison of the peak soft X-ray and $3.6 \mathrm{~cm}$ radio luminosity of solar and stellar flares. The upper limits derived from these preflare observations are shown by red arrows. Two peaks in the early main flare phase are indicated with a lowercase red $x$, the main peak with an uppercase $X$. We also show nanoflares in quiet regions (Krucker and Benz, 2000), microflares in active regions (Benz et al., 1981; Fürst, Benz, and Hirth, 1982; Gary, Hartl, and Shimizu, 1997), impulsive and gradual flares (Benz and Güdel, 1994), quiescent BY Dra, dMe, and dKe stars (Güdel et al., 1993), and a long-duration flare on the dMe star EQ Peg (Kundu et al., 1988). The dashed line presents the relation given in Equation 1. (Graphics adapted from Benz and Güdel, 2010, and based on Krucker and Benz, 2000.) 